\title{
Partial genome assembly of the medicinal plant Ephedra sinica
}

\author{
Qiushi Li, Jeremy S. Morris, Peter J. Facchini, Sam Yeaman* \\ Department of Biological Sciences, University of Calgary, Calgary, AB, T2N 1N4, Canada
}

*To whom correspondence should be addressed. E-mail: samuel.yeaman@ucalgary.ca

\begin{abstract}
Ephedra sinica is a high-value medicinal plant that produces important phenylpropylamino alkaloids pseudoephedrine and ephedrine. Few genomics resources exist for $E$. sinica, which has been characterized as a tetraploid with a monoploid genome size of $8.56 \mathrm{~Gb}$. Here we reported a partial genome assembly of $E$. sinica $(12.8 \mathrm{~Gb})$ based on Illumina short-read sequencing at low coverage.
\end{abstract}

\section{Background}

Transcript and genomic resources of even moderate quality have greatly accelerate the isolation of enzymes and the elucidation of biosynthetic pathways leading to a variety of plant specialized metabolites. Reconstitution of these pathways in industrially tractable hosts ,in particular microorganisms, is a key step toward a 'greener' and more socially conscious pharmaceutical industry.

Ephedra sinica is a 'living fossil' plant that produces the phenylpropylamino alkaloids pseudoephedrine and ephedrine, the former of which is a widely marketed decongestant and the latter is recognized as an essential medicine by the World Health Organization ${ }^{[2]}$. Despite the importance of these medicines, their biosynthesis has not been fully elucidated. The commercial production of (pseudo)ephedrine is largely based on a chemical synthesis process $^{[3]}$. However, one key industrial biotransformation step is performed using yeast fermentation, which results in the production of the chiral precursor $(R)$-phenylacetylcarbinol. Given that bioreactors designed to grow yeast cells at industrial scale (i.e. $>1000 \mathrm{~L}$ ), and the affiliated separation chemistry systems are already integrated into the manufacturing process, fully biosynthetic production of (pseudo)ephedrine from inexpensive starting materials could be viewed as a compelling business case ${ }^{[4]}$.

Recently, the enzyme that catalyzes the ultimate step in (pseudo)ephedrine biosynthesis in $E$. sinica, phenylalkylamine $\mathrm{N}$-methyltransferase (EsPaNMT), was cloned and shown to function in microorganisms ${ }^{[5]}$.Although progress towards complete microbial biosynthesis is being achieved by substituting the antecedent biosynthetic steps with functionally analogous enzymes from other organisms, yields remain low. In E. sinica, at least four (pseudo)ephedrine biosynthetic enzymes, as well as an unknown number of transport and accessory proteins, remain to be discovered. As repeatedly demonstrated in various microbial biosynthesis projects to date, implementation of these plant proteins is key to enhancing metabolite yield to commercially viable levels ${ }^{[6-9]}$. 
Aside from (pseudo)ephedrine, EsPaNMT was also shown to produce a series of phenethylamine and tryptamine psychedelics. Notably, these molecules are currently attracting significant interest from the pharmaceutical industry due to wide-ranging medicinal benefits ${ }^{[10]}$. Given the wide range of useful activities displayed by EsPaNMT, it is reasonable to suspect that other E. sinica enzymes and biosynthetic proteins may have similar properties which could enable microbial biosynthesis of these other emerging classes of medicinal compounds.

Few genomics resources exist for $E$. sinica, which has been characterized as a tetraploid with a monoploid genome size of $1 \mathrm{Cx}$-value=8.75 pg $(8.56 \mathrm{~Gb})^{[11]}$. Here we reported a partial genome assembly of $E$. sinica based on Illumina short-read sequencing at low coverage.

\section{Materials \& Methods}

The specimen of $E$. sinica used in this study was grown from open-pollinated seed acquired from wild populations originating in northern China (Horizon Herbs LLC). Total genomic DNA was extracted from 3 grams of one-year old stem material using a standard CTAB modified with the addition of RNase A treatment and polysaccharide removal ${ }^{[5,12]} .800 \mu \mathrm{g}$ total DNA of greater than $>20 \mathrm{~kb}$ in length was obtained (A260/A280 $=2.0, A 260 / A 230=2.1$ ) (Figure 1).

For shotgun sequencing on Illumina HiSeq X, we constructed four PCR-Free libraries with insert-size of $550 \mathrm{bp}$, and obtained 349 million $150 \mathrm{bp}$ pair-ended reads (base count of 105.5 $\mathrm{Gb}$, monoploid coverage of $12 \mathrm{x}$ ).

We first filtered the raw Illumina data with fastp $\left.{ }^{[13]}(-q) 20-u \quad 40-\mid 51\right)$ before performing shortreads based assembly. We chose the memory-efficient de novo assembler SOAPdenovo2 ${ }^{[14]}$, which was designed for large genomes, in consideration of the genome size of $E$. sinica. SOAPdenovo-63mer module of the SOAPdenovo2 was used for the contig level assembly with the best kmer-size of 25 . The same dataset was used for the scaffolding step.

\section{Results}

The statistics of the assembly results are shown in Table 1.

Table 1. Summary of the genome assembly of $E$. sinica

\begin{tabular}{l|l|l}
\hline & Contig level & Scaffold level \\
\hline Assembled length with Ns & $12,131,244,078 \mathrm{bp}$ & $12,754,467,985 \mathrm{bp}$ \\
Assembled length without Ns & $12,131,244,078 \mathrm{bp}$ & $11,911,939,172 \mathrm{bp}$ \\
Number of Sequences & $39,810,445$ & $36,316,713$ \\
Longest sequence & $11,479 \mathrm{bp}$ & $18,185 \mathrm{bp}$ \\
Length N50 & $348 \mathrm{bp}$ & $446 \mathrm{bp}$ \\
\hline
\end{tabular}

The GC content of the assembled E. sinica genome is $30.62 \%$. A total of $6,494,909$ contigs were successfully placed in scaffolds. The BUSCO ${ }^{[15]}$ (v4.1.4) assessment of completeness of the contig level assembly against the database of eukaryota_odb10 shows that $96.5 \%$ of the 
total 255 BUSCO genes are absent in the assembly. Among the nine assembled BUSCO genes, two genes (pre-mRNA-splicing factor ISY1 homolog and sm-like protein LSM3A) are complete and presented as single copy.

\section{Discussion and Conclusions}

De novo assembly of large genomes, such as E. sinica, is highly complex and resourceintensive due to the high proportion of repetitive DNA and polyploidy. Proper practices are usually based either on high sequencing depth of short reads ${ }^{[16]}$ or long-read sequencing ${ }^{[17]}$, which can be expensive. Despite a more comprehensive approach, an effort to sequence the related Ephedra equisetina genome yielded results comparable to those reported here ${ }^{[16]}$.

Our endeavor provides a rough image of the $E$. sinica genome for the research community. We hope that increasingly advanced sequencing and assembly technologies lead to a more complete genome in the near future. Aside from the biotechnological value of genomics-guided gene discovery discussed earlier; the study of Ephedra spp. has also been suggested to be valuable from an evolutionary developmental biology perspective. As recently reviewed, it is an ideal gymnosperm lineage to investigate innovations including pollination, seed dispersal and adaptation to extreme environments ${ }^{[18]}$.

\section{Data availability}

The raw Illumina sequencing data was deposited on NCBI genbank under BioProject: PRJNA734610. The assembled contig and scaffold level of the $E$. sinica genome sequences were deposited on Dryad under resource-id 105514.

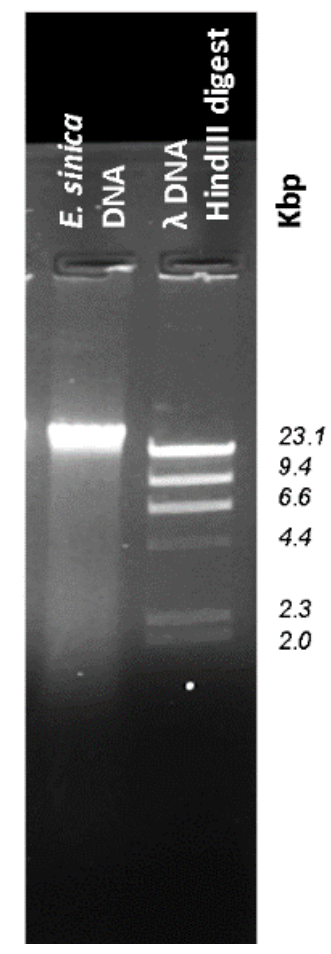

Figure 1. Agarose gel electrophoresis analysis of extracted DNA. Hindlll-digested lambda phage DNA is used as a high molecular weight standard. DNA is stained with ethidium bromide and visualized with UV light. 


\section{Bibliography}

[1] M. Yamazaki, A. Rai, N. Yoshimoto, K. Saito, Perspective: functional genomics towards new biotechnology in medicinal plants. Plant Biotechnol. Rep. 2018, 12, 69-75.

[2] World Health Organization, Ment. Holist. Heal. Some Int. Perspect. 2019, 21, 119-134.

[3] J. M. Hagel, R. Krizevski, F. Marsolais, E. Lewinsohn, P. J. Facchini, Biosynthesis of amphetamine analogs in plants. Trends Plant Sci. 2012, 17, 404-412.

[4] V. Uppada, K. Satpute, P. K. Agarwal, G. A. Swaminathan, S. B. Noronha, Metabolic Engineering of Saccharomyces cerevisiae for Synthesis of Ephedrine Alkaloids. in Curr. Dev. Biotechnol. Bioeng. Funct. Genomics Metab. Eng., Elsevier, 2016, pp. 269-290.

[5] J. S. Morris, R. A. Groves, J. M. Hagel, P. J. Facchini, An N-methyltransferase from Ephedra sinica catalyzing the formation of ephedrine and pseudoephedrine enables microbial phenylalkylamine production. J. Biol. Chem. 2018, 293, 13364-13376.

[6] M. Dastmalchi, L. Chang, R. Chen, L. Yu, X. Chen, J. M. Hagel, P. J. Facchini, Purine Permease-Type Benzylisoquinoline Alkaloid Transporters in Opium Poppy. Plant Physiol. 2019, 181, 916-933.

[7] M. Dastmalchi, X. Chen, J. M. Hagel, L. Chang, R. Chen, S. Ramasamy, S. Yeaman, P. $\mathrm{J}$. Facchini, Neopinone isomerase is involved in codeine and morphine biosynthesis in opium poppy. Nat. Chem. Biol. 2019, 15, 384-390.

[8] X. Chen, J. M. Hagel, L. Chang, J. E. Tucker, S. A. Shiigi, Y. Yelpaala, H.-Y. Chen, R. Estrada, J. Colbeck, M. Enquist-Newman, A. B. Ibáñez, G. Cottarel, G. M. Vidanes, P. J. Facchini, A pathogenesis-related 10 protein catalyzes the final step in thebaine biosynthesis. Nat. Chem. Biol. 2018, 14, 738-743.

[9] B. R. Lichman, G. T. Godden, J. P. Hamilton, L. Palmer, M. O. Kamileen, D. Zhao, B. Vaillancourt, J. C. Wood, M. Sun, T. J. Kinser, L. K. Henry, C. Rodriguez-Lopez, N. Dudareva, D. E. Soltis, P. S. Soltis, C. R. Buell, S. E. O'Connor, The evolutionary origins of the cat attractant nepetalactone in catnip. Sci. Adv. 2020, 6, eaba0721.

[10] R. G. dos Santos, J. C. Bouso, J. M. Rocha, G. N. Rossi, J. E. Hallak, The Use of Classic Hallucinogens/Psychedelics in a Therapeutic Context: Healthcare Policy Opportunities and Challenges. Risk Manag. Healthc. Policy 2021, Volume 14, 901-910.

[11] S. M. Ickert-Bond, A. Sousa, Y. Min, I. Loera, J. Metzgar, J. Pellicer, O. Hidalgo, I. J. Leitch, Polyploidy in gymnosperms-Insights into the genomic and evolutionary consequences of polyploidy in Ephedra. Mol. Phylogenet. Evol. 2020, 147, 106786.

[12] V. Dos Reis Falcão, A. Pedroso Tonon, M. Cabral Oliveira, P. Colepicolo, RNA Isolation method for polysaccharide rich algae: agar producing Gracilaria tenuistipitata (Rhodophyta). J. Appl. Phycol. 2008, 20, 9-12.

[13] S. Chen, Y. Zhou, Y. Chen, J. Gu, fastp: an ultra-fast all-in-one FASTQ preprocessor. Bioinformatics 2018, 34, i884-i890.

[14] R. Luo, B. Liu, Y. Xie, Z. Li, W. Huang, J. Yuan, G. He, Y. Chen, Q. Pan, Y. Liu, J. Tang, G. Wu, H. Zhang, Y. Shi, Y. Liu, C. Yu, B. Wang, Y. Lu, C. Han, D. W. Cheung, S.-M. Yiu, S. Peng, Z. Xiaoqian, G. Liu, X. Liao, Y. Li, H. Yang, J. Wang, T.-W. Lam, J. Wang, SOAPdenovo2: an empirically improved memory-efficient short-read de novo assembler. Gigascience 2012, 1, DOI 10.1186/2047-217X-1-18.

[15] M. Seppey, M. Manni, E. M. Zdobnov, BUSCO: assessing genome assembly and annotation completeness. Methods Mol. Biol. 2019, 1962, 227-245.

[16] T. Wan, Z.-M. Liu, L.-F. Li, A. R. Leitch, I. J. Leitch, R. Lohaus, Z.-J. Liu, H.-P. Xin, Y.-B. Gong, Y. Liu, W.-C. Wang, L.-Y. Chen, Y. Yang, L. J. Kelly, J. Yang, J.-L. Huang, Z. Li, P. Liu, L. Zhang, H.-M. Liu, H. Wang, S.-H. Deng, M. Liu, J. Li, L. Ma, Y. Liu, Y. Lei, W. Xu, L.-Q. Wu, F. Liu, Q. Ma, X.-R. Yu, Z. Jiang, G.-Q. Zhang, S.-H. Li, R.-Q. Li, S.-Z. Zhang, Q.-F. Wang, Y. Van de Peer, J.-B. Zhang, X.-M. Wang, A genome for gnetophytes and early evolution of seed plants. Nat. Plants 2018, 4, 82-89. 
bioRxiv preprint doi: https://doi.org/10.1101/2021.06.02.446745; this version posted June 2, 2021. The copyright holder for this preprint (which was not certified by peer review) is the author/funder. All rights reserved. No reuse allowed without permission.

[17] Pacific Biosciences of California, "Go Big or Go Home - Tackling a Giant Genome," can be found under https://www.pacb.com/blog/tackling-a-giant-genome/

[18] V. S. Di Stilio, S. M. Ickert-Bond, Ephedra as a gymnosperm evo-devo model lineage. Evol. Dev. 2021, 1-11. 